2. To: (Receiving Organization)

Distribution

5. Proj./Prog./Dept./Div.:

Tank 241-TX-113/Waste

Management/TB\&P/Process

Engineering

8. Originator Remarks:

This document is being released into the supporting document

system for retrievability purposes.

3. From: (Originating Organization) Technicai Basis and Planning

6. Design Authority/ Design Agent/Cog. Engr.:

Dennis J. McCain

4. Related EDT No.:

7. Purchase Order No.:

$N / A$

11: Receiver Remarks: '..... -11A: Design Baseline Document? [] Yes [X] No For release.

9. Equip./Component No.: $\mathrm{N} / \mathrm{A}$

10. System/Bldg./Facility: $241-T X-113$

12. Major Assm. Dwg. No.: N/A

13. Permit/Permit Application No.: N/A

14. Required Response Date: $07 / 30 / 98$

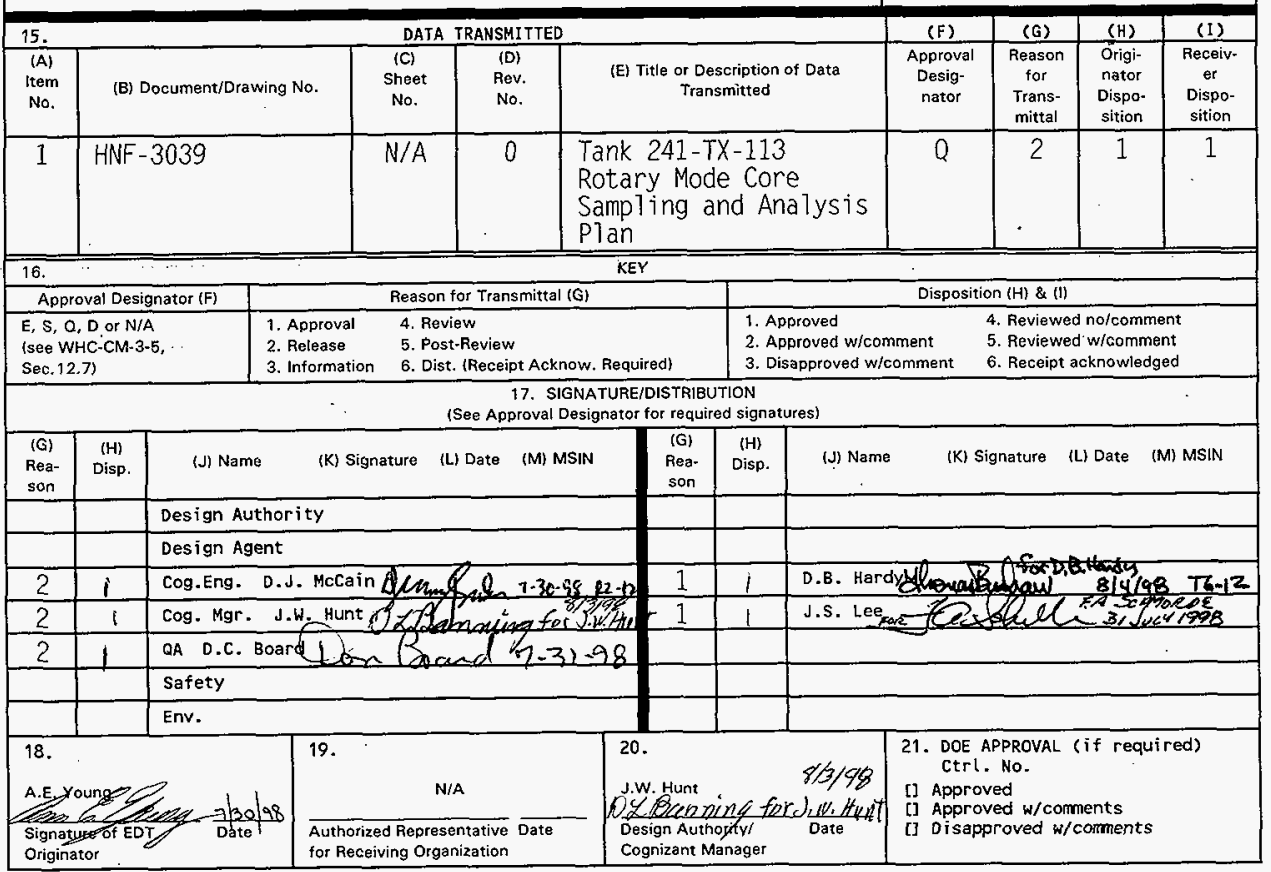

BD-7400-172-2 (05/96) GEF097 


\title{
Tank 241-TX-113 Rotary Mode Core Sampling and Analysis Plan
}

Dennis J. McCain

Lockheed Martin Hanford, Corp. , Richland, WA 99352

U.S. Department of Energy Contract DE-AC06-96RL13200

\author{
EDT/ECN: EDT-622460 UC: 2070 \\ Org Code: 7 A110 Charge Code: N4G2C \\ B\&R Code: EW 3120074 Total Pages:2y
}

Key Words: Tank 241-TX-113, 241-TX-113, TX-113, TX Farm, Rotary Mode. Rotary, Core, Sampling, Analysis, Plan

Abstract: $\quad N / A$

TRADEMARK DISCLAIMER. Reference herein to any specific comercial product, process, or service by trade name, trademark, manufacturer, or otherwise, does not necessarily constitute or imply its endorsement, recommendation, or favoring by the United States Government or any agency thereof or its contractors or subcontractors.

Printed in the United States of America. To obtain copies of this document, contact: Document Control Services, P.O. Box 950, Maílstop H6-08, Richland WA 99352, Phone (509) 372-2420; Fax (509) 376-4989.
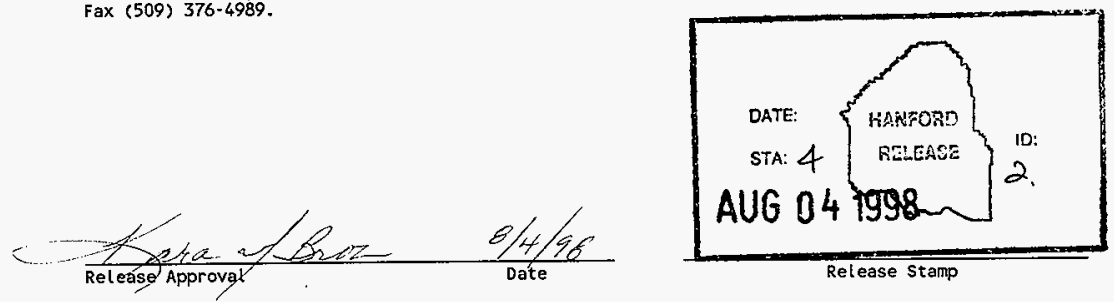

Approved for Public Release 


\title{
Tank 241-TX-113 \\ Rotary Mode Core Sampling and Analysis Plan
}

\author{
D. J. McCain \\ Lockheed Martin Hanford Corporation
}

Prepared for the U.S. Department of Energy

Office of Environmental Restoration

and Waste Management 
HNF-3039, Rev. 0

\section{TABLE OF CONTENTS}

1.0 SAMPLING AND ANALYSIS OBJECTIVES $\ldots \ldots \ldots \ldots \ldots \ldots \ldots \ldots \ldots \ldots$

2.0 SAMPLING EVENT REQUIREMENTS $\ldots \ldots \ldots \ldots \ldots \ldots \ldots \ldots \ldots \ldots \ldots \ldots$

3.0 LABORATORY ANALYSIS REQUIREMENTS $\ldots \ldots \ldots \ldots \ldots \ldots \ldots \ldots \ldots \ldots \ldots \ldots \ldots \ldots \ldots \ldots \ldots$

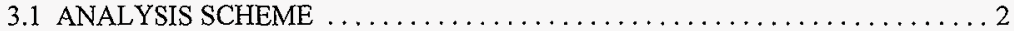

3.2 SPECIFIC METHODS AND ANALYSES $\ldots \ldots \ldots \ldots \ldots \ldots \ldots \ldots \ldots \ldots$

3.3 INSUFFICIENT SEGMENT RECOVERY $\ldots \ldots \ldots \ldots \ldots \ldots \ldots \ldots \ldots \ldots$

4.0 QUALITY ASSURANCE AND QUALITY CONTROL $\ldots \ldots \ldots \ldots \ldots \ldots \ldots \ldots$

4.1 LABORATORY OPERATIONS $\ldots \ldots \ldots \ldots \ldots \ldots \ldots \ldots \ldots \ldots \ldots \ldots \ldots$

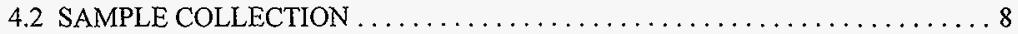

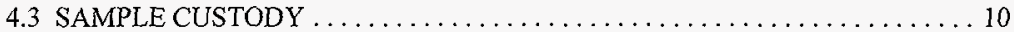

5.0 EXCEPTIONS, CLARIFICATIONS, AND ASSUMPTIONS $\ldots \ldots \ldots \ldots \ldots \ldots \ldots 11$

5.1 EXCEPTIONS TO DQO REQUIREMENTS $\ldots \ldots \ldots \ldots \ldots \ldots \ldots \ldots \ldots \ldots \ldots$

5.2 CLARIFICATIONS AND ASSUMPTIONS $\ldots \ldots \ldots \ldots \ldots \ldots \ldots \ldots \ldots$

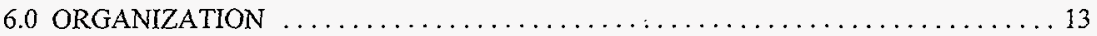

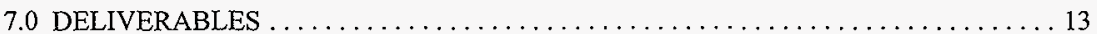

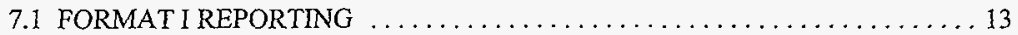

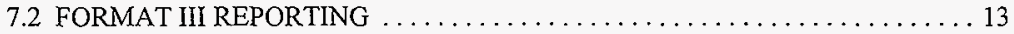

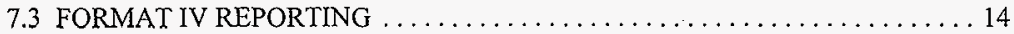

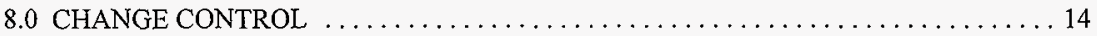

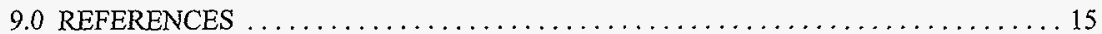

APPENDIX A: EXPECTED PHYSICAL PROFILE OF TANK TX-113 CORE

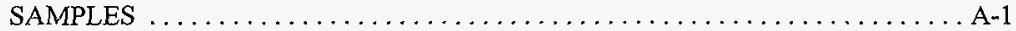

\section{LIST OF TABLES}

1: Tank TX-113 Chemical, Radiological, and Physical Analytical Requirements: Solids . . . 4

2: Tank TX-113 Chemical, Radiological, and Physical Analytical Requirements: Liquids . . . 6

3: QC Precision and Accuracy Requirements for the Analyses . . . . . . . . . . . . . . 9

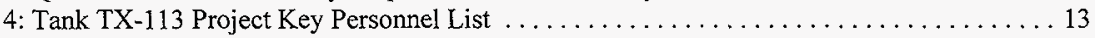

A-1: Tank 241-TX-113 Physical Profile Estimate Riser $3 \ldots \ldots \ldots \ldots \ldots \ldots \ldots \ldots$ A-2

A-2: Tank 241-TX-113 Physical Profile Estimate Riser $5 \ldots \ldots \ldots \ldots \ldots \ldots \ldots \ldots$ A-3

\section{LIST OF FIGURES}

A-1: Tank 241-TX-113 Physical Profile Estimate (Riser 3) . . . . . . . . . . . . A-2

A-2: Tank 241-TX-113 Physical Profile Estimate (Riser 5 ) ................... A-3 
HNF-3039, Rev. 0

\section{LIST OF ABBREVIATIONS}

\begin{tabular}{ll} 
Ci & curie \\
CPO & Characterization Project Operations \\
DQO & data quality objective \\
DSC & differential scanning calorimetry \\
GEA & gamma energy analysis \\
g & gram \\
g/L & gram per liter \\
IC & ion chromatography \\
ICP/AES & inductively coupled plasma - atomic emission spectroscopy \\
kgal & kilogallon \\
kL & kiloliter \\
L & liter \\
LFL & lower flammability limit \\
LiBr & lithium bromide \\
LMHC & Lockheed Martin Hanford Corporation \\
$\mathrm{mL}$ & milliliter \\
MSL & mean sea level \\
PIC & person in charge \\
PNNL & Pacific Northwest National Laboratory \\
PRSST & Propagating Reactive System Screening Tool \\
QA & quality assurance \\
QC & quality control \\
RSST & Reactive System Screening Tool \\
SAP & sampling and analysis plan \\
TGA & thermogravimetric analysis \\
TIC & total inorganic carbon \\
TOC & total organic carbon \\
TWRS & Tank Waste Remediation System \\
TX-113 & 241-TX-113 \\
WMH & Waste Management Hanford \\
$\mu \mathrm{Ci}$ & microcurie \\
$\mu \mathrm{Ci} / \mathrm{g}$ & microcurie per gram \\
$\mu \mathrm{g}$ C/g & micrograms of carbon per gram \\
$\mu \mathrm{g} / \mathrm{g}$ & micrograms per gram \\
$\mu \mathrm{g} / \mathrm{mL}$ & micrograms per milliiter \\
& \\
\hline
\end{tabular}




\subsection{SAMPLING AND ANALYSIS OBJECTIVES}

This sampling and analysis plan (SAP) identifies characterization objectives pertaining to sample collection, laboratory analytical evaluation, and reporting requirements for push mode core samples from tank 241-TX-113 (TX-113). It is written in accordance with Tank Safety Screening Data Quality Objective (Dukelow et al. 1995), Data Quality Objective to Support Resolution of the Flammable Gas Safety Issue (Bauer 1998) and the Historical Model Evaluation Data Requirements (Simpson and McCain 1997). The Tank Characterization Technical Sampling Basis document (Brown et al. 1998) also identifies Retrieval, Pretreatment and Immobilization as an issue that applies to tank TX-113. As a result, a 150 gram composite of solids shall be made and archived for that program.

\subsection{SAMPLING EVENT REQUIREMENTS}

As of April 30, 1998, tank TX-113 contained a total waste volume of approximately $2,300 \mathrm{~kL}$, (607 kgal), consisting entirely of saltcake with $60.6 \mathrm{~kL}$ (16 kgal) of drainable liquid (Hanlon 1998). This waste volume is equivalent to 5.80 meters ( 228 inches) of waste as measured from the centerline of the tank. A physical profile prediction based on waste fill history and previous sampling information is provided in Appendix A. Historical data suggests Al-clad sludge is part of the tank waste (Place 1997).

Prior to core sampling, the dome space (below the riser) shall be measured for the presence of flammable gases. The measurement shall be taken from within the dome space and the data reported as a percentage of the lower flammability limit (LFL). The results shall be transmitted to the tank coordinator within ten working days of the sampling event (Schreiber 1998). If the dome space results are above 10 percent of the LFL when analyzing with a combustible gas meter or above 25 percent of the LFL when analyzing by gas chromatography/mass spectrometry or gas-specific monitoring, the necessity for recurring sampling for the flammable gas concentration and the frequency of such sampling will be determined by the Flammable Gas Safety Project.

During core sampling, the drill string will be monitored for flammable gas. If the monitoring instrument in the drill string indicates a level greater than $10 \%$ of the LFL during intrusive activities, then a vapor grab sample shall be taken and sent to Pacific Northwest National Laboratory (PNNL) for analysis (Bauer 1998). Any additional vapor sampling is not within the scope of this SAP.

Tank TX-113 will be rotary mode core sampled using a rotary mode core sampling system. Two core samples, each consisting of 13 segments, are expected to be taken from risers 3 and 5 . The sampling objective is to obtain two vertical profiles of the waste; therefore, more or fewer segments may need to be taken depending on the accuracy of the current waste volume records in comparison to presampling zip cord readings.

Universal samplers will be used for these samples. If quality-affecting changes to the sampling requirements must be made (including the risers, sampling truck, or segments to be sampled), the 
change must be recorded and approved by the cognizant engineer and tank coordinator before sampling. This information may be recorded on a permanent data sheet or recorded directly in sampling work packages WS-98-00072 (riser 3) and WS-98-00073 (riser 5). These work packages contain the operating procedures and the chain-of-custody records for this sampling event.

One field blank for tank TX-113 shall be obtained in accordance with procedure TO-060-003. The Characterization Project Operations (CPO) person in charge (PIC) or the PIC designate will verify that the field blank is properly created and shipped. For sampling events having multiple PICs, CPO shall determine which PIC will be responsible for the field blank. This field blank is to accompany the samples to the laboratory. All collected samples shall be shipped to the laboratory following the Load/Transport Sample Cask(s) procedure (TO-080-090). Core samples should be transported to the laboratory within three calendar days from the time each segment is removed from the tank.

If lithium bromide ( $\mathrm{LiBr}$ ) solution is used in the collection of the core samples, it should be a 0.3 \pm 0.01 molar solution with a pH greater than 8 . Characterization Project Operations must state the batch number and amount of fluid added at each segment. This information should be indicated on the chain-of-custody form that accompanies the sample to the laboratory. A sample of the $\mathrm{LiBr}$ solution must be provided to the laboratory. This sample shall consist of a container filled with $\mathrm{LiBr}$ solution from the same batch of $\mathrm{LiBr}$ solution used during the sampling. This solution shall be analyzed for lithium and bromide in order to determine the concentration of the tracer at the time the sample was taken. If analysis of the waste samples indicates contamination by the $\mathrm{LiBr}$ solution, these data will be used to determine the amount of contamination. If more than one batch of $\mathrm{LiBr}$ solution is used during sampling event, one solution sample must be provided for each batch in addition to the field blank.

\subsection{LABORATORY ANALYSIS REQUIREMENTS}

\subsection{ANALYSIS SCHEME}

In order to comply with Dukeiow et al. (1995), Bauer (1998), and Simpson and McCain (1997), the following steps shall be performed on each sample:

- Extrude segments, videotaping the extrusion and photographing the extruded segments. The extrusion procedure is LO-160-103 at the 222-S Laboratory.

- $\quad$ Filter or centrifuge drainable liquids as needed prior to analysis.

- Separate solids into half-segments and homogenize.

- Form stratum composite samples and core composite samples from each core per tank coordinator direction.

- Analyze drainable liquids, homogenized half-segment subsamples, stratum composite, and core composite samples from each core as shown in Tables 1 and 2.

- Form a 150 gram solid composite sample from one (or both) cores and archive this material for the Retrieval, Pretreatment, and Immobilization Program.

- Archive all remaining material. 
If liner liquid is observed during extrusion and the liquid is in sufficient quantity to collect, the liner liquid may be retained and analyzed at the discretion of the tank coordinator. 'In this event, this addition of analyses may not require a revision to this SAP.

\section{For core samples from tank TX-113, the shipping container must be vented every 27 days to release any accumulated gas.}

Opportunistic analyses as defined in Kristofzski (1996) are to be included when the laboratory is not operating at maximum capacity. Any decisions, observations, or deviations made to this work plan, or during the sample breakdown and analyses shall be documented in writing with justification. These decisions and observations shall be reported in the data report. The reporting formats for analyses are contained in Tables 1 and 2 and are described in Section 7.0.

\subsection{SPECIFIC METHODS AND ANALYSES}

The analyses in Tables 1 and 2 to be performed on tank TX-113 core samples are based on the safety screening data quality objective (DQO), the flammable gas DQO, and the historical model DQO referenced in Section 1.0. The laboratory procedure numbers which shall be used for the analyses are included in the tables. Sample preparation procedures that may be used at the 222-S Laboratory are LA-549-141 for fusion digestion, LA-505-159 or LA-505-163 for acid digestion of samples, and LA-504-101 for water leach of solids.

The historical model DQO requires phased analysis periods, and selection of optimal segments for full suite analysis. The laboratory is to notify the tank coordinator once primary analyses are complete for each segment.

\subsection{INSUFFICIENT SEGMENT RECOVERY}

If the amount of material recovered from samples taken from a tank is insufficient to perform the analyses requested in the $\mathrm{SAP}$ and permit a minimum $10 \mathrm{~mL}$ archive per sample, the laboratory shall notify the tank coordinator within one working day. At that time, a prioritization of the analyses may be provided to the laboratory. Any analyses prescribed by the SAP, but not performed, shall be identified in the appropriate data report with justification provided for nonperformance. 
HNF-3039, REV. 0

Table 1: Tank TX-113 Chemical, Radiological, and Physical Analytical Requirements: Solids (2 Sheets)

\begin{tabular}{|c|c|c|c|c|c|c|c|c|c|c|c|c|c|c|c|}
\hline \multicolumn{16}{|c|}{ SOLID ANALYSES } \\
\hline \multicolumn{2}{|l|}{ Project Name } & \multicolumn{3}{|c|}{ TX-113 Rotary Mode Core Sample } & \multicolumn{8}{|c|}{ COMMENTS } & \multicolumn{3}{|c|}{ REPORTING LEVELS } \\
\hline \multicolumn{2}{|c|}{ Plan Number } & \multicolumn{3}{|c|}{ HNF-3039, Rev. 0} & \multirow{4}{*}{\multicolumn{8}{|c|}{$\begin{array}{l}\text { Homogenization Test - Per Laboratory Discretion } \\
\text { Field Blank - Required } \\
\text { Hot Cell Blank - Per Laboratory Discretion } \\
\text { LiBr Solution Blank - Required if LiBr solution is used }\end{array}$}} & FORMAT I & \multicolumn{2}{|c|}{ Immediate Notification } \\
\hline \multicolumn{2}{|c|}{ PROGRAM } & \multicolumn{3}{|c|}{ PROGRAM CONTACTS } & & & & & & & & & FORMAT II & \multicolumn{2}{|l|}{ Process Control } \\
\hline \multicolumn{2}{|c|}{ A. Safety Screening } & TWRS Safety & \multicolumn{2}{|l|}{ R. J. Cash } & & & & & & & & & FORMAT III & \multicolumn{2}{|l|}{ Safety Screening } \\
\hline \multicolumn{2}{|c|}{ B. Historical Data } & TWRS Proc. Eng. & \multicolumn{2}{|l|}{ B. C. Simpson } & & & & & & & & & FORMAT IV & \multicolumn{2}{|l|}{ Waste Management } \\
\hline \multicolumn{2}{|c|}{ C. Process Control } & TWRS Proc. Eng. & \multicolumn{2}{|l|}{ D. J. MeCain } & \multicolumn{4}{|c|}{ TANK } & \multicolumn{4}{|c|}{ \#CORES } & FORMAT V & \multicolumn{2}{|l|}{ RCRA Compliance } \\
\hline \multicolumn{2}{|c|}{ D. Flammable Gas } & TWRS Safety & R. E. Bauer & & \multicolumn{3}{|c|}{$T X-113$} & & \multicolumn{3}{|c|}{2} & & FORMAT VI & Special & \\
\hline & & RUMARY ANAXYSES & & & SAMPLE & & & QU. & ALITY CC & ONTRO & & & CRITERIA & & \\
\hline PROGRAM & METHOD & ANALYSIS & PROCEDURE & $\begin{array}{c}1 / 2 \text { SEG } \\
\text { SLDG } \\
\text { SC }\end{array}$ & \begin{tabular}{|l|} 
STRAT \\
/COMP \\
\end{tabular} & COMP & PREP ${ }^{2}$ & DUP & \$PIKE & BLK & STD & UNITS & $\begin{array}{c}\text { NOTIFICATTON } \\
\text { LMMT }^{4}\end{array}$ & $\begin{array}{l}\text { EXPECTED } \\
\text { RANGE }\end{array}$ & FORMAT \\
\hline$A, B$ & DSC & Energy & $\begin{array}{l}\text { LA-514-113 } \\
\text { LA-514-114 }\end{array}$ & $x$ & & $\mathrm{x}$ & d & ea smpl & $\mathrm{N} / \mathrm{A}$ & N/A & $\operatorname{ca} A B$ & $J^{\prime} / \mathrm{g}$ & $\geq 480^{3,6}$ & unknown & $\mathrm{I}, \mathrm{MI}, \mathrm{TV}$ \\
\hline $\mathrm{A}, \mathrm{B}, \mathrm{D}$ & TGA & $\% \mathrm{H}_{2} \mathrm{O}$ & LA-514-114 & $\bar{x}$ & $x$ & $x$ & $d$ & ea smpl & N/A & $N / A$ & ea $A B$ & wit\% & none & 63.2 & III, IV \\
\hline $\mathrm{A}, \mathrm{B}, \mathrm{D}$ & Gravimetry & Bulk density & LO-160-103 & $x^{15}$ & $x$ & $x$ & $d$ & $\mathrm{~N} / \mathrm{A}$ & $\mathrm{N} / \mathrm{A}$ & N/A & N/A & $\mathrm{g} / \mathrm{mL}$ & none & 1.41 & III, IV \\
\hline A, B & Atpha counuing & Tocal Alpha & LA.508.101 & $\mathrm{x}^{15}$ & & $x$ & for $a$ & ca smpl & $1 / \mathrm{mtrx}$ & $\mathrm{ca} \mathrm{PB}$ & ea $A B$ & $\mu \mathrm{Ci} / \mathrm{g}$ & $>41^{6}$ & unknown & I, III, IV \\
\hline$B, D$ & Beta counting & ${ }^{30} \mathrm{Sr}$ & LA-220-101 & $x^{7}$ & $x$ & $x$ & for a & ca smpl & $1 / \mathrm{murx}^{8}$ & $\mathrm{ca} \mathrm{PB}$ & $\mathrm{ca} A \mathrm{AB}$ & $\mu \mathrm{Ci} / \mathrm{g}$ & none & 81 & IV \\
\hline $\mathrm{B}, \mathrm{D}$ & GEA & ${ }^{137} \mathrm{Cs}^{9}$ & LA-548-121 & $x$ & $x$ & $x$ & f & ca smpl & N/A & \begin{tabular}{|l|} 
ca PBB \\
\end{tabular} & ca $A B$ & $\mu \mathrm{Ci} / g$ & nonc & 189 & IV \\
\hline D & $\begin{array}{l}\text { Acidification } \\
\text { Persulfate oxidation }\end{array}$ & TIC & LA. $342-100$ & & $x$ & & d & ea smpl & $1 / \mathrm{mtrx}$ & $\mathrm{caAB}$ & $\mathrm{AB}$ & $\mu \mathrm{g} C / \mathrm{g}$ & TOC $>45,000^{5.5}$ & unknown & I, IV \\
\hline$A, C$ & ICP/AES & $\begin{array}{l}\mathrm{Li}, \mathrm{Al}, \mathrm{Cr}, \mathrm{FC}, \mathrm{Mn}, \mathrm{Na} \\
\mathrm{Ni}, \mathrm{Si}, \mathrm{U}, 11\end{array}$ & $\begin{array}{l}\text { LA-505-151 } \\
\text { LA-505-161 }\end{array}$ & $x$ & & & for a & ca smpl & $1 / \mathrm{murx} x^{20}$ & $\mathrm{CaAB}$ & ea $A B$ & $\mu \mathrm{g} / \mathrm{g}$ & none & varied & III, IV \\
\hline B & ICP/AES & $\mathrm{Na}, \mathrm{Al}, \mathrm{Cr}^{16}$ & $\begin{array}{l}\text { LA-505-151 } \\
\text { LA-505-161 }\end{array}$ & & & $x$ & for a & ca smipl & $1 / \mathrm{mtrx} x^{20}$ & ca $A B$ & $\mathrm{Ca} \mathrm{AB}$ & $\mu \mathrm{g} / \mathrm{g}$ & none & Varied & IV \\
\hline D & ICP/AES & $\begin{array}{l}\mathrm{Ca}, \mathrm{Fe}, \mathrm{K}, \mathrm{Ni}, \mathrm{Al}, \mathrm{Ba} \\
\mathrm{Si}, \mathrm{B}, \mathrm{Bi}, \mathrm{Na}, \mathrm{Zr}, \mathrm{Zn}^{17}\end{array}$ & $\begin{array}{l}\text { LA-505-151 } \\
\text { LA-505-161 }\end{array}$ & & $x$ & & for a & ca smpl & $1 / \mathrm{mtr} x^{20}$ & ca $\mathrm{AB}$ & $\mathrm{CA} A \mathrm{AB}$ & $\mu g / g$ & none & varied & IV \\
\hline B & ICP/AES & $\mathrm{Na}, \mathrm{Al}, \mathrm{Cr}^{16}$ & $\begin{array}{l}\text { LA-505-151 } \\
\text { LA-505-161 }\end{array}$ & $x^{7}$ & & $x$ & $w$ & ea smpl & $1 / \max ^{20}$ & $\mathrm{ca} \mathrm{AB}$ & $\mathrm{Ca} \mathrm{AB}$ & $\mu g / g$ & none & Varied & $\mathrm{rV}$ \\
\hline c & IC & $\mathrm{Br}^{10}$ & LA-533-105 & $\mathrm{x}$ & & & $w$ & ca smpl & $1 / \mathrm{mtrx}$ & ea $A B$ & ea $\mathrm{AB}$ & $\mu \mathrm{g} / \mathrm{mL}$ & none & varied & IV \\
\hline $\mathrm{B}$ & IC & $\mathrm{NO}_{3}, \mathrm{SO}_{4}{ }^{18}$ & LA-533-105 & & & $x$ & $\mathrm{w}$ & ea smpl & $1 /$ metrx & ea $A B$ & ea $\mathrm{AB}$ & $\mu g / \mathrm{mL}$ & none & varied & IV \\
\hline D & IC & $\begin{array}{l}\text { formate, oxalate, } \mathrm{NO}_{2} \text {, } \\
\mathrm{PO}_{4}, \mathrm{Cl}, \mathrm{F}^{19}\end{array}$ & LA-533-105 & & $\mathrm{x}$ & & $\mathbf{w}$ & ea smpl & $1 /$ mtrx & $\operatorname{cas} A B$ & ea $\mathrm{AB}$ & $\mu \mathrm{g} / \mathrm{mL}$ & none & varied & NV \\
\hline $\mathrm{B}, \mathrm{D}$ & Persulfate & TOC & LA-342-100 & $x^{7}$ & $x$ & $x$ & $\mathrm{~d}$ & ea smpl & 1/mux & ea $A \bar{B}$ & ea $A B$ & $\mu \mathrm{g} \mathrm{C} / \mathrm{g}$ & $>45,000^{5,5}$ & 15,900 & $\mathrm{I}, \mathrm{IV}$ \\
\hline $\mathrm{B}$ & Beta countifg & Total Beta & LA-508-101 & $x^{3}$ & & $\mathrm{x}$ & for a & ea smp1 & $1 / \mathrm{mtrx}$ & ea $A B$ & $\mathrm{ea} \mathrm{AB}$ & $\mu \mathrm{Cl} / \mathrm{g}$ & none & unknown & IV \\
\hline $\mathrm{B}$ & $\begin{array}{l}\text { Kinctic } \\
\text { phosphorescence }\end{array}$ & Uranium & LA-925-009 & $x^{7}$ & & $\mathrm{x}$ & for a & ea smpl & $1 / \mathrm{m}$ trx & $\mathrm{caAB}$ & ea $\mathrm{AB}$ & $\mu g / g$ & none & 1,180 & IV \\
\hline$D$ & Tiuation & $\mathrm{OH}$ & LA-211-102 & & $x$ & & $w$ & ea smpl & $1 /$ max & $\mathrm{ea} A \mathrm{~B}$ & $\mathrm{ea} A \mathrm{~B}$ & $\mu \mathrm{g} / \mathrm{g}$ & none & 125,000 & IN \\
\hline D & Spectrophotometry & $\mathrm{Cr}(\mathrm{vI})$ & LA-265-101 & & $x$ & & $w$ & ea smol & 1/mitrx & $\mathrm{ea} A B$ & ea $\mathrm{AB}$ & $\mu \mathrm{g} / \mathrm{g}$ & none & 2,570 & IV \\
\hline D & Gravimetry & Setuling behavior & $\sec ^{22}$ & $\mathrm{x}$ & & & $d$ & ea smpl & N/A & $\mathrm{N} / \mathrm{A}$ & N/A & $\mathrm{cm} / \mathrm{hr}$ & none & unknown & IV \\
\hline D & Centrifuge & Vol\% solids & LA- $519-132$ & $\mathrm{x}$ & & & d & N/A & N/A & N/A & N/A & vol\% & none & unknown & IV \\
\hline
\end{tabular}


Table 1: Tank TX-113 Chemical, Radiological, and Physical Analytical Requirements: Solids (2 Sheets)

\begin{tabular}{|c|c|c|c|c|c|c|c|c|c|c|c|c|c|c|c|}
\hline \multirow[b]{2}{*}{ PROGRAM } & \multicolumn{3}{|c|}{ SECONDARY ANALXSES } & \multicolumn{4}{|c|}{ SAMPLE ${ }^{1}$} & \multicolumn{4}{|c|}{ QUALITY CONKROX ${ }^{3}$} & \multicolumn{3}{|c|}{ CRITERIA } & \multirow[b]{2}{*}{ FORMAT } \\
\hline & METHOD & ANALYSIS & PROCEDURE & $\begin{array}{c}1 / 2 \text { SEG } \\
\text { SLDG } \\
\text { SC } \\
\end{array}$ & $\begin{array}{l}\text { STRAT } \\
\text { /COMP }\end{array}$ & COMP & PREP $^{2}$ & DUP & SPIKE & BLK & STD & UNTTS & $\begin{array}{c}\text { NOTIFICATION } \\
\text { LMMIT }^{4}\end{array}$ & $\begin{array}{c}\text { EXPECTED } \\
\text { RANGE* }\end{array}$ & \\
\hline A & Alpha counting & ${ }^{299 / 240} \mathrm{Pu}^{13}$ & LA-953-104 & $x$ & & & $\mathrm{f}$ & ea smpl & $1 / \mathrm{mtrx}^{8}$ & ea PB & $\mathrm{ea} \mathrm{AB}$ & $\mu \mathrm{Ci} / \mathrm{g}$ & none & 0.064 & iII, IV \\
\hline $\mathrm{A}$ & PRSST & propagating behavior ${ }^{\text {in }}$ & LT-510-103 & $x$ & & & $d$ & N/A & N/A & N/A & $\operatorname{seg}^{2 t}$ & $\mathrm{~J} / \mathrm{g}$ & none & unknown & IV \\
\hline A & Fumace Oxidation & $\operatorname{Toc}^{14}$ & LA-344-105 & $x^{\top}$ & & & $w$ & ea smpl & $1 /$ matrx & ea $A B$ & ea AB & $\mu \mathrm{g} \mathrm{C} / \mathrm{g}$ & $>45,000^{5,6}$ & 15,900 & $\mathrm{I}, \mathrm{III}, \mathrm{rV}$ \\
\hline
\end{tabular}

${ }^{i / 2}$ SEG SLDG/SC $=1 / 2$ segment, studge or saltcake; STRAT/COMP = scratum subsample or composite COMP =Composite for Historical DQO

${ }^{2} \mathrm{~d}=$ direct, $\mathrm{f}=$ fusion, $\mathrm{a}=$ acid, $\mathrm{w}=$ water

${ }^{3} \mathrm{DUP}=$ duplicate, $\mathrm{BLK}=$ blank, $\mathrm{STD}=$ calibration standard, ea $=$ each, smpl $=$ sample, $\mathrm{AB}=$ analytical batch, $\mathrm{PB}=$ preparation blank, merx $=$ matrix, $\mathrm{N} / \mathrm{A}=\mathrm{not}$ applicable

"Units for notification limits and expected range are those listed in the "units" column.

'Dry weight basis

${ }^{6}$ These analytes are to be compared to the linit by calculating the one-sided, upper $95 \%$ confidenee limit for the sample result (to be perforned by Process Engineering).

${ }^{1}$ These analyses wjll be performed on one half-segment per corc, as directed by the tank coordinator.

${ }^{8}$ Tracer or carrier may be used in place of a spike and results corrected for recovery.

'Results should be reported for all GEA analytes, however the QC requirements of Table 3 apply to ${ }^{13} \mathrm{Cs}$.

${ }^{10} \mathrm{Br}$ is required to correct \% $\mathrm{H}_{2} \mathrm{O}$ measurement (to be performed by Process Engineering).

Results should be repored for all IC analytes. However, the QC requirements of 'Table 3 only apply to thase identified in this talble.

${ }^{1 \mathrm{~L}} \mathrm{\textrm {i }}$ is required to correct \% $\mathrm{H}_{2} \mathrm{O}$ measurement (to be performed by Process Enginecring). $\mathrm{Al}, \mathrm{Cr}, \mathrm{Fe}, \mathrm{Mn}, \mathrm{Na}, \mathrm{Ni}$, Si, and $\mathrm{U}$ are required as secondary safety screening analytes,

to be performed if total alpha activity excecds notification limit.

Results should be reported for all ICP/AES analytes; however, the QC requirements of Table 3 apply only to those identified herc.

${ }^{12}$ No procedure is available for the sctuling rate test. Work will be performed to an approved test plan, which will be referenced in une data package.

${ }^{13}$ Analysis to be perforned if tolal alpha activity limit is excecded.

${ }^{14}$ TOCJPRSST is requircd only on the subscgment with the highest result (other than bistorical DQO optimum scgment) if the sample's DSC result excceds $480 \mathrm{~J} / \mathrm{g}$.

${ }^{15}$ These analyses requircd as a primary analysis only on the lower half-segment of each segment. If the half-scgment selected for additional historical DQO analyses is an

upper half-segment, they should be done on this sample as well.

${ }^{16} \mathrm{Na}, \mathrm{Al}, \mathrm{Cr}$ are fingepprint antlytes for historical. Results should be reported for all ICP/AES anslytes; however, the QC requirements of Table 3 apply only to those identified herc.

${ }^{17} \mathrm{Ca}, \mathrm{Fe}, \mathrm{K}, \mathrm{Ni}, \mathrm{Zn}, \mathrm{Al}, \mathrm{Ba}, \mathrm{Si}, \mathrm{B}, \mathrm{Bi}, \mathrm{Na}, \mathrm{Zn}$, and $\mathrm{ZI}$ are from the flammable gas $\mathrm{DQO}$, and are required only on the stratum samples. Results should be reported for all ICP/AES analytes;

however, the QC requirements of Table 3 apply only to those identified here.

${ }^{13}$ Nitrate and Sulfate are fingerprint analytes for historical DQO. Results shoutd be reported for all IC analytes. Howcver, the QC requircments of Table 3 onfy apply to thosc identified in this table.

${ }^{19}$ Formate, oxalate, nitrite, phosphate, chloride, and fluoride are required only on the flammable gas stratum samples. Results should be reported for all IC analytes.

However, the QC requirements of Table 3 only apply to those identified in this table.

${ }^{20}$ Either serial dilutions or matrix spikes wil be performed.

${ }^{21}$ Calibration of heater resistance, time, temperature, pressure, containment volume, and sample weight will be performed to measure accuracy as described in procedurc LT-510-103. 
Table 2: Tank TX-113 Chemical, Radiological, and Physical Analytical Requirements: Liquids (2 Sheets)

\begin{tabular}{|c|c|c|c|c|c|c|c|c|c|c|c|c|c|c|}
\hline \multirow{2}{*}{\multicolumn{2}{|c|}{ Project Name }} & \multirow{2}{*}{\multicolumn{10}{|c|}{$\begin{array}{l}\text { LIQUID ANALYSES } \\
\end{array}$}} & \multirow{2}{*}{\multicolumn{3}{|c|}{ REPORTING LEVELS }} \\
\hline & & \multicolumn{2}{|c|}{ TX-113 Rotary Mode Core Sample } & & & & & & & & & & & \\
\hline \multicolumn{2}{|c|}{ Plan Number } & & & & & & \multicolumn{5}{|c|}{\begin{tabular}{|c|} 
COMMENTS \\
Homogenization Test - Per Laboratory Discretion
\end{tabular}} & \begin{tabular}{|l|} 
FORMAT I \\
\end{tabular} & \multicolumn{2}{|c|}{ Inmediate Notification } \\
\hline \multicolumn{2}{|c|}{ PROGRAM } & \multicolumn{5}{|c|}{ PROGRAM CONTACTS } & \multirow{2}{*}{\multicolumn{5}{|c|}{$\begin{array}{l}\text { Field Blank - Required } \\
\text { Hot Cell Blank - Per Laboratory Discretion }\end{array}$}} & FORMAT II & \multicolumn{2}{|c|}{$\begin{array}{l}\text { Process Control } \\
\end{array}$} \\
\hline \multicolumn{2}{|c|}{ A. Safety Screening } & TWRS Safcty & \multicolumn{4}{|c|}{ R. J. Cash } & & & & & & FORMAT III & \multicolumn{2}{|c|}{ Safety Screening } \\
\hline B. Process Cont & & TWRS Proc. Eng. & \multicolumn{4}{|l|}{ D. J. McCain } & \multicolumn{5}{|c|}{$\begin{array}{l}\text { Hot Cell Blank - Per Laboratory Discretion } \\
\text { LiBr Solution Blank - Required if HHF is used }\end{array}$} & FORMAT IV & \multicolumn{2}{|c|}{ Waste Maragement } \\
\hline \multicolumn{2}{|c|}{ C. Flammable Gas } & TWRS Safety & \multicolumn{4}{|l|}{ R. E. Barer } & \multicolumn{2}{|c|}{ TANK } & \multicolumn{3}{|c|}{ \#CORES } & FORMAT V & \multicolumn{2}{|c|}{ RCRA Compliance } \\
\hline & & & & & & & \multicolumn{2}{|c|}{$\mathrm{TX}-113$} & \multicolumn{3}{|c|}{2} & FORMAT VI & Special & \\
\hline & \multicolumn{3}{|c|}{ PRIMARY ANALYSES } & \multicolumn{3}{|c|}{ SAMP $^{1}$} & \multicolumn{4}{|c|}{ OUALITY CONTROL ${ }^{3}$} & & CRITERIA & & \\
\hline PROGRAM & METHOD & ANALYSIS & PROCEDURE & $\begin{array}{l}\text { FB \& } \\
\text { SEG } \\
\text { LIO } \\
\end{array}$ & STRAT & PREP $^{2}$ & DUP & SPIKE & BLK & STD & UNITS & $\begin{array}{l}\text { NOTIFICATION } \\
\text { LIMIT }^{4}\end{array}$ & $\begin{array}{l}\text { EXPECTED } \\
\text { RANGE }^{4}\end{array}$ & FORMAT \\
\hline A & Dsc & Energy & $\begin{array}{l}\text { LA-514-113 } \\
\text { LA-514-114 } \\
\end{array}$ & $x$ & & d & ea smpl & $\mathrm{N} / \mathrm{A}$ & $\mathrm{N} / \mathrm{A}$ & ca $\mathrm{AB}$ & $\mathrm{J} / \mathrm{g}$ & $\geq 480^{3,6}$ & unknown & I, III, rV \\
\hline$\overline{A, C}$ & TGA & $\% \mathrm{H}_{2} \mathrm{O}$ & LA-514-114 & $x$ & $x$ & d & ea smpl & N/A & N/A & $\mathrm{CaAB}$ & $\mathrm{wt} \%$ & none & 45.9 & III, IV \\
\hline$A$ & Alpha counting & Total Alpha & LA-508-101 & $x$ & & $d$ & ea smpl & $1 /$ mtrx & ea PB & $e A A B$ & $\mu \mathrm{Ci} / \mathrm{mL}$ & $>61.5^{6}$ & unknown & I, III, IV \\
\hline$A, C$ & Gravimetry & Specific Gravity & LA-510-112 & $x$ & $\mathrm{x}$ & $d$ & ea smpl & $\mathrm{N} / \mathrm{A}$ & $\mathrm{N} / \mathrm{A}$ & ca $A B$ & none & none & 1.43 & IV \\
\hline$A, B, C$ & ICPIAES & $\begin{array}{l}\mathrm{Li}, \mathrm{Al}, \mathrm{Cr}, \mathrm{Fc}, \mathrm{Mn}, \\
\mathrm{Na}, \mathrm{Ni}, \mathrm{Si}, \mathrm{U}, \mathrm{Ca}, \mathrm{K} \\
\mathrm{Zn}, \mathrm{Zr}, \mathrm{Ba}, \mathrm{B}, \mathrm{Bi}{ }^{7} \\
\end{array}$ & $\begin{array}{l}\text { LA-505-151 } \\
\text { LA-505-161 }\end{array}$ & $\mathrm{x}$ & $\mathrm{x}$ & $d^{8}$ & ea smpl & $1 / \mathrm{mtrx}{ }^{2}$ & ea $P B$ & $\mathrm{caAB}$ & $\mu \mathrm{g} / \mathrm{mL}$ & none & varied & III, N \\
\hline C & Ion selective elcctrode & Ammonia ${ }^{17}$ & LA-631-001 & $x$ & & $d^{8}$ & ea smpl & $1 /$ tank & ea $A B$ & ca $A B$ & $\mu \mathrm{g} / \mathrm{mL}^{12}$ & none & 968 & IV \\
\hline$B, C$ & IC & $\begin{array}{l}\mathrm{Br} \text {, formate, oxalate, } \\
\mathrm{NO}_{3}, \mathrm{NO}_{2}, \mathrm{PO}_{4}, \mathrm{SO}_{4} \\
\mathrm{Cl} \text {, and } \mathrm{F}^{11}\end{array}$ & LA-533-105 & $\mathrm{x}$ & $x$ & $d^{8}$ & ea smpl & $1 / \mathrm{mtrx}$ & ca $A B$ & ca $A B$ & $\mu \mathrm{g} / \mathrm{mL}$ & none & varied & IV \\
\hline c & \begin{tabular}{|l} 
Acidification' \\
Persulfate
\end{tabular} & TIC/TOC & LA-342-100 & & $\mathrm{x}$ & d & ca smpl & $1 /$ mtrx & ea $A B$ & ea $A B$ & $\mu \mathrm{g} \mathrm{C} / \mathrm{mL}$ & TOC $>45,000^{5,6,10}$ & unknown & I, IV \\
\hline $\mathrm{A}$ & Visual & Organic & LA-519-151 & $x$ & & d & $\mathrm{N} / \mathrm{A}$ & $\mathrm{N} / \mathrm{A}$ & $\mathrm{N} / \mathrm{A}$ & $\mathrm{N} / \mathrm{A}$ & $\mathrm{N} / \mathrm{A}$ & presence & none & I, III, IV \\
\hline $\mathrm{C}$ & Titration & $\mathrm{OH}$ & LA-211-102 & & $\bar{x}$ & d & ea smpl & $1 / \mathrm{mtax}$ & ea $\mathrm{AB}$ & ca $\mathrm{AB}$ & $\mu \mathrm{g} / \mathrm{mL}$ & none & 73,500 & IV \\
\hline $\mathrm{c}$ & Spectrophotometry & Cr (VI) & LA-265-101 & & $\mathrm{x}$ & $\mathrm{d}$ & ca smpl & $1 / \operatorname{mtrx}$ & ea $\mathrm{AB}$ & ea $\mathrm{AB}$ & $\mu \mathrm{g} / \mathrm{mL}$ & none & 3,050 & IV \\
\hline C & GEA & ${ }^{137} \mathrm{Cs}^{19}$ & LA-548-121 & & $x$ & d & ea smpl & $1 / \mathrm{mtrx}^{14}$ & $\mathrm{ea} A \mathrm{~B}$ & ea $\mathrm{AB}$ & $\mu \mathrm{Ci} / \mathrm{mL}$ & none & 152 & IV \\
\hline C & beta counting & ${ }^{96} \mathrm{Sr}$ & LA-220-101 & & $x$ & $d$ & ea smpl & $1 / m$ trex & ea $\mathrm{AB}$ & $\mathrm{eaB}$ & $\mu \mathrm{Ci} / \mathrm{mL}$ & none & 53.8 & IV \\
\hline $\mathrm{C}$ & gravimetric & Settling behavior & $\operatorname{see}^{15}$ & $\mathrm{x}$ & & $d$ & ea smpl & N/A & $\mathrm{N} / \mathrm{A}$ & N/A & $\mathrm{cm} / \mathrm{hr}$ & none & unknown & IV \\
\hline c & centrifuge & solids fraction & LA-519-132 & $x$ & & d & N/A & N/A & N/A & $\mathrm{N} / \mathrm{A}$ & vol\% & none & unknown & IV \\
\hline & SECC & ONDARY ANALYSES & & $\mathrm{SA}$ & $\mathrm{MP}^{1}$ & & & UALITY C & ONTRO & & & CRITERIA & & \\
\hline PROGRAM & METHOD & ANALYSIS & PROCEDURE & $\begin{array}{l}\text { SEG } \\
\text { LIQ } \\
\end{array}$ & & PREP $^{2}$ & DUP & SPIKE & BLK & STD & UNITS & $\begin{array}{l}\text { NOTIFICATION } \\
\text { LIMIT }^{+} \\
\end{array}$ & $\begin{array}{l}\text { EXPECTED } \\
\text { RANGE } \\
\end{array}$ & FORMAT \\
\hline$A$ & Alpha counting & ${ }^{239 / 40} \mathrm{Pu}^{13}$ & LA-953-104 & $x$ & & $d$ & ea smpl & $1 / \mathrm{mtrx}^{14}$ & ea $A B$ & $\mathrm{ea} A B$ & $\mu \mathrm{Ci} / \mathrm{mL}$ & none & 0.02 & III, $\mathrm{NV}$ \\
\hline $\mathrm{A}$ & PRSST & propagating behavior ${ }^{18}$ & LT-510-103 & $x$ & & $d$ & N/A & N/A & $\mathrm{N} / \mathrm{A}$ & $\sec ^{22}$ & $\mathrm{~J} / \mathrm{g}$ & none & unknown & IV \\
\hline $\mathrm{A}$ & Furnace Oxidation & $\operatorname{TOC}^{10}$ & LA-344-105 & $x$ & & $d$ & ea smpl & $1 / \mathrm{mtrx}$ & ea $A B$ & ea $A B$ & $\mu \mathrm{g} \mathrm{C} / \mathrm{mL}$ & $>45,000^{5,6,16}$ & unknown & I, III, IV \\
\hline
\end{tabular}




\section{HNF-3039, REV. 0}

Table 2: Tank TX-113 Chemical, Radiological, and Physical Analytical Requirements: Liquids (2 Sheets)

'FB = field blank, SEG LIQ = segment-level drainable liquid, STRAT = stratum or composite liquid

${ }^{2} d=$ direct

${ }^{3}$ DUP $=$ duplicate, $\mathrm{BLK}=$ blank, $\mathrm{STD}=$ calibration standard, ea $=$ each, smpl $=$ sample, $\mathrm{AB}=$ analytical batch, $\mathrm{PB}=$ preparation blank, mtrx $=$ matrix, $\mathrm{N} / \mathrm{A}=$ not applicable

"Units for notification limits and expected range arc those listed in the "units" column.

${ }^{5}$ Dry weight basis

"These analytes are to be compared to the limit by calculating the one-sided, upper $95 \%$ confidence limit for the sample result (to be performed by Process Engineering)

${ }^{\prime} \mathrm{Li}$ is required to correct $\% \mathrm{H}_{2} \mathrm{O}$ measurement (to be performed by Process Engineering). $\mathrm{Al}, \mathrm{Cr}, \mathrm{Fe}, \mathrm{Mn}, \mathrm{Na}, \mathrm{Ni}, \mathrm{Si}$, and $\mathrm{U}$ are required as secondary safety screening analytes; required if total alpha exceeds

notification limits. The remainder are for the flammable gas DQO. Results should be reported for all ICP/AES analytes, however, the QC reguirements of Table 3 apply only to those identified here.

${ }^{8}$ Direct liquid samples may be diluted in acid or water to adjust to proper sample size and/or $\mathrm{pH}$.

${ }^{9}$ Either serial dilutions or matrix spikes will be perforned.

${ }^{10} \mathrm{TOC}$ by the furnace oxidation method is performed on a sample if the sample's DSC result exceeds $480 \mathrm{~J} / \mathrm{g}$.

${ }^{11} \mathrm{Br}$ is required to correct \% $\mathrm{H}_{2} \mathrm{O}$ mcasurement (to be performed by Process Engineering). The remainder are required for the flammable gas DQO.

Results should be reported for all IC analytes, however, the QC requirements of Table 3 apply only to those listed.

${ }^{12}$ Micrograms anmonia per milliliter of as-received sample.

${ }^{13}$ Performed if total alpha cxcceds notification limit.

${ }^{14}$ Tracer or carrier may be used in place of a spike and results corrected for recovery.

${ }^{15}$ No procedure is available for the sctlling rate test. Work will be performed to an approved test plan, which will be referenced in the data package.

${ }^{16}$ Corrected from weight basis to volumetric basis assuming a liquid density of $1.0 \mathrm{~g} / \mathrm{mL}$

${ }^{17}$ Ammonia analysis should be performcd as rapidly as possible following subsampling to minimize volatilization.

${ }^{18}$ Calibration of heater resistatice, time, tempcrature, pressure, containment volume, and sample wejght will be performed to mcasure accuracy as described in procedure LT-510-103.

${ }^{19}$ Results should be reported for all GEA analytes, however, the QC requirements of Table 3 apply only to ${ }^{137} \mathrm{Cs}$.

${ }^{20}$ No procedure is avaitable for the settling rate test. Work will be performed to an approved test plan, which will be referenced in the data package. 


\subsection{QUALITY ASSURANCE AND QUALITY CONTROL}

Processes, services, activities, and conditions adverse to quality which do not conform to requirements specified in this SAP or references herein shall be controlled to prevent inadvertent use. Nonconforming sampling and analysis processes shall be identified, controlled, reported, and dispositioned as required by the Nonconformance Item Reporting and Control (PHMC 1997).

\subsection{LABORATORY OPERATIONS}

Laboratories performing analyses in support of this SAP shall have approved and implemented Quality Assurance (QA) Plans. These QA plans shall meet the Hanford Analytical Services Quality Assurance Requirements Document (DOE 1997) minimum requirements as the baseline for laboratory quality systems. The 222-S Laboratory Quality Assurance Plan (Markel 1997) specifies the requirements for assuring the quality of sample analysis conducted at the 222-S Laboratory. Quality requirements for conducting Characterization Project sampling and analysis are described in Tank Waste Remediation System Characterization Project, Quality Policies (Board 1997) and this SAP. Characterization Project sampling and analysis shall be conducted in conformance with these requirements.

Analytical quality control (QC) requirements (duplicates, spikes, blanks, laboratory control samples) are identified in Tables 1 through 3 . The laboratory shall also use calibration and calibration check standards appropriate for the analytical instrumentation being used (see DOE [1997] for definitions of QC samples and standards). The criteria presented are goals for demonstrating reliable method performance. It is understood that the laboratory will follow its internal QC system for required actions whenever QC failures occur. If sample QC failures occur or if all analyses cannot be performed (e.g., insufficient sample), analysts shall consult with supervisors/customers to determine the proper action. The laboratory should provide a suggested course of action at that time. All sample QC failures and limitations on the associated data shall be discussed in the narrative of the data report. Proper notification of all data not meeting QC requirements shall be included with the data.

\subsection{SAMPLE COLLECTION}

Before sampling can be performed on a tank, available risers must be identified for use in the sampling event. The selected risers must be inspected and prepared to confirm their ability to be used in sampling. Safety hazards must be identified and special precautions must be taken if needed. If deemed necessary by the sampling cognizant engineers and tank coordinator, video surveillance should be performed to identify any potential problems that may occur during the sampling event.

Samples are to be taken from a tank and shipped to the performing laboratory by CPO in accordance with the respective work package(s). The chain-of-custody form for this work package shall identify samples by a unique number and state the type of sampler used (retained gas sampler or universal sampler) for each sample before being shipped to the 222-S Laboratory. Approved procedure TO-080-090 [Load/Transport Sample Cask(s)] is to be used during the 
sampling event. Pertinent sampling information (e.g. unusual waste characteristics, $\mathrm{x}$-ray scan results, $\mathrm{LiBr}$ solution used, or detecting debris) should be noted in the comment section of the chain-of-custody form.

Characterization Project Operations should transport each sample collected to the performing laboratory within 3 calendar days of removing the sample from the tank. A verbal notification by $\mathrm{CPO}$ is to be made to the 222-S Laboratory at 373-2435 at least 24 hours in advance of an expected shipment.

Table 3: QC Precision and Accuracy Requirements for the Analyses

\begin{tabular}{|c|c|c|c|c|}
\hline Analysis/Method & $\begin{array}{l}\text { Duplicate } \\
\text { Criteria } \\
\text { (RPD) }\end{array}$ & $\begin{array}{l}\text { Spike Criteria } \\
(\% \text { recovery })^{2}\end{array}$ & $\begin{array}{l}\text { Preparation } \\
\text { Blank Criteria }\end{array}$ & $\begin{array}{l}\text { L CSCriteria } \\
\text { (\% recovery) }\end{array}$ \\
\hline \multicolumn{5}{|l|}{ Solids } \\
\hline DSC & $\leq 30$ & N/A & N/A & $80-120$ \\
\hline TGA & $\leq 30$ & N/A & N/A & $80-120$ \\
\hline Bulk density & N/A & N/A & $\mathrm{N} / \mathrm{A}$ & N/A \\
\hline Total Uranium & $\leq 20$ & $75-125$ & $<\mathrm{EQL}$ & $80-120$ \\
\hline Total alpha & $\leq 20$ & $75-125$ & $<\mathrm{MDA}$ & $70-130$ \\
\hline Total beta & $\leq 20$ & $75-125$ & $<\mathrm{MDA}$ & $80-120$ \\
\hline ICP/AES & $\leq 20$ & $75-125$ & $<$ EQL & $80-120$ \\
\hline PRSST $^{6}$ & N/A & N/A & N/A & $\mathrm{N} / \mathrm{A}$ \\
\hline IC & $\leq 20$ & $75-125$ & $<\mathrm{EQL}$ & $80-120$ \\
\hline TOC/TIC & $\leq 20$ & $75-125$ & $<E Q L$ & $80-120$ \\
\hline${ }^{90} \mathrm{Sr}$ & $\leq 20$ & $75-125^{5}$ & $<\mathrm{MDA}$ & $80-120$ \\
\hline GEA & $\leq 20$ & N/A & $<\mathrm{MDA}$ & $80-120$ \\
\hline${ }^{239 / 240} \mathrm{Pu}$ & $\leq 20$ & $75-125^{5}$ & $<\mathrm{MDA}$ & $70-130$ \\
\hline $\mathrm{Cr}(\mathrm{VI})$ & $\leq 20$ & $75-125$ & $<\mathrm{EQL}$ & $80-120$ \\
\hline $\mathrm{OH}$ & $\leq 10$ & $75-125$ & $<\mathrm{EQL}$ & $90-110$ \\
\hline Settling Rate & $\leq 10$ & N/A & N/A & $\mathrm{N} / \mathrm{A}$ \\
\hline Solids Fraction & N/A & N/A & N/A & N/A \\
\hline
\end{tabular}


HNF-3039, Rev. 0

\begin{tabular}{|c|c|c|c|c|}
\hline Analysis/Method & $\begin{array}{l}\text { Duplicate } \\
\text { Criteria } \\
\text { (RPD) }\end{array}$ & $\begin{array}{l}\text { Spike Criteria } \\
(\% \text { recovery })^{2}\end{array}$ & $\begin{array}{l}\text { Preparation } \\
\text { Blank Criteria }\end{array}$ & $\begin{array}{l}\text { LCS criteria } \\
(\% \text { recovery })\end{array}$ \\
\hline \multicolumn{5}{|l|}{ Liquids } \\
\hline $\mathrm{DSC}$ & $\leq 20$ & N/A & N/A & $80-120$ \\
\hline TGA & $\leq 20$ & $\mathrm{~N} / \mathrm{A}$ & $\mathrm{N} / \mathrm{A}$ & $80-120$ \\
\hline Specific gravity & $\leq 20$ & $N / A$ & $\mathrm{~N} / \mathrm{A}$ & N/A \\
\hline Total alpha & $\leq 20$ & $75-125$ & $<\mathrm{MDA}$ & $70-130$ \\
\hline ICP/AES & $\leq 20$ & $75 \cdot 125$ & $<\mathrm{EQL}$ & $80-120$ \\
\hline PRSST $^{6}$ & N/A & N/A & N/A & N/A \\
\hline IC & $\leq 20$ & $75-125$ & $<\mathrm{EQL}$ & $80-120$ \\
\hline Ammonia & $\leq 10$ & $75-125$ & $<\mathrm{EQL}$ & $90-110$ \\
\hline TIC/TOC & $\leq 20$ & $75-125$ & $<\mathrm{EQL}$ & $80-120$ \\
\hline $\mathrm{OH}$ & $\leq 10$ & $75-125$ & $<\mathrm{EQL}$ & $90-110$ \\
\hline $\mathrm{Cr}$ (VI) & $\leq 20$ & $75-125$ & $<\mathrm{EQL}$ & $80-120$ \\
\hline GEA & $\leq 20$ & N/A & $<\mathrm{MDA}$ & $80-120$ \\
\hline${ }^{239 / 240} \mathrm{Pu}$ & $\leq 20$ & $75-125^{5}$ & $<\mathrm{MDA}$ & $70-130$ \\
\hline${ }^{90} \mathrm{Sr}$ & $\leq 20$ & $75-125^{5}$ & $<\mathrm{MDA}$ & $80-120$ \\
\hline Settling Rate & $\leq 10$ & $\mathrm{~N} / \mathrm{A}$ & $\mathrm{N} / \mathrm{A}$ & N/A \\
\hline Solids Fraction & N/A & N/A & N/A & N/A \\
\hline
\end{tabular}

Notes:

$\begin{array}{ll}\text { N/A } & =\text { not applicable } \\ \text { MDA } & =\text { minimum detectable activity } \\ \text { EQL } & =\text { estimated quantitation limit } \\ \text { LCS } & =\text { laboratory control standard }\end{array}$

'For the calculation of the relative percent difference (RPD), both the sample and duplicate results must exceed the $\mathrm{EQL}$ or MDA. Failures are permissible if the requirements in the QA section are followed.

${ }^{2}$ The criteria are recommended. Failures are permissible if the requirements in the QA section are followed.

${ }^{3}$ When a blank exceeds the EQL or MDA, sample results that exceed the contribution from the blank twenty-fold or more are reportable. See also the QA section of this SAP.

${ }^{4}$ For some analyses, this could be a method spike or a blank spike. Ranges are percent recovery of theoretical.

${ }^{5}$ A tracer or carrier may be substituted for the spike.

${ }^{6}$ See Section 5.1. 


\subsection{SAMPLE CUSTODY}

The chain-of-custody form is initiated by the sampling team as described in the work package. Samples are shipped in a cask and sealed with a Waste Tank Sample Seal (see below).

\begin{tabular}{||l|l||}
\hline \multicolumn{2}{|c||}{ WASTE TANK SAMPLE SEAL } \\
\hline Supervisor: & Sample No.: \\
\hline Date of Sampling: & Time of Sampling: \\
\hline Shipment No.: & Serial No.: \\
\hline
\end{tabular}

Each sample number shall be created using the sample's core and segment number. For instance, segment 1 of core 197 would be sample number 197-01. The sealed and labeled samples are shipped to the laboratory along with the chain-of-custody form. The receipt and control of samples in the 222-S Laboratory are described in laboratory procedure LO-090-101.

\subsection{EXCEPTIONS, CLARIFICATIONS, AND ASSUMPTIONS}

\subsection{EXCEPTIONS TO DQO REQUIREMENTS}

The safety screening DQO (Dukelow et al. 1995) states that cyanide analysis is required when the energetics notification limit is exceeded and the total organic carbon (TOC) is less than its notification limit. Because the ferrocyanide safety issue has been closed, cyanide analysis is no longer required as a secondary analyte under the safety screening DQO (Cash 1997).

Analysis by the Reactive System Screening Tool (RSST) method is currently required as a secondary analysis by the safety screening DQO. However, the Propagating Reactive System Screening Tool (PRSST) analysis is now preferred over the RSST testing. As such, the RSST requirement in Tables 1 and 2 has been removed replaced by a PRSST requirement.

\subsection{CLARIFICATIONS AND ASSUMPTIONS}

A number of clarifications and assumptions relating to the notification limits or decision thresholds identified in the applicable DQO efforts need to be made with respect to the analyses in Tables 1 and 2. Each of these issues is discussed below:

- Any exothermic reaction determined by differential scanning calorimetry (DSC) must be reported on a dry weight basis as shown in equation (1) using the weight percent water determined from thermogravimetric analysis (TGA).

$$
\text { Exotherm }(\text { dry } w t)=\frac{[\text { exotherm }(\text { wet } w t) \times 100]}{\left(100-\frac{o}{2} \text { water }\right)}
$$


NOTE: $\quad$ A large error in the DSC dry weight basis value may result when the wt $\%$ water value is greater than 90 percent water, dry weight basis, however, this conversion is still required.

- The safety screening DQO (Dukelow et al. 1995) requires that additional analyses be performed if total alpha activity is greater than $1 \mathrm{~g} / \mathrm{L}$. For solids, total alpha activity is measured in $\mu \mathrm{Ci} / \mathrm{g}$ rather than $\mathrm{g} / \mathrm{L}$. To convert the notification limit for total alpha activity into a number more readily usable by the laboratory, it was assumed that all alpha decay originates from $\mathrm{Pu}-239$. The notification limit may then be calculated as shown in equation (2):

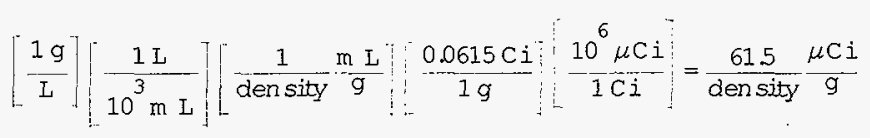

NOTE: Solid samples measured for total alpha activity shall also be measured for density. The notification limit for solid subsamples shall be $41 \mu \mathrm{Ci} / \mathrm{g}$ until the density is measured. At such time, the notification limit will be adjusted according to equation 2 and the total alpha activity results will be reevaluated against the new limit.

- The safety screening DQO (Dukelow et al. 1995) states that the analytical results should be compared to their notification limits at a $95 \%$ confidence level (one-tailed test). The equation for determining the upper confidence value is shown in equation 3.

$$
\hat{\mu}+t_{(n-1)} * \frac{\sqrt{a^{2}}}{\sqrt{n}}
$$

Where $\mu$ is the sample mean, $\sigma^{2}$ is the sample variance, $n$ is the number of observations (for a sample run in duplicate, $n$ equals 2 ), and $t_{(n-1)}$ is the quantile from Student's $t$ distribution with (n-1) degrees of freedom (for a one-sided $95 \%$ confidence interval and when $\mathrm{n}$ is $2, \mathrm{t}$ is 6.314). This equation is appropriate for confidence limit estimates of the mean when the sample size is small. This equation, as well as a table of values for the Student $t$ statistic, should be found in any introductory statistics textbook (e.g., Lapin 1983).

The laboratory is requested to report all analytical results recovered from the inductively coupled plasma - atomic emission spectroscopy (ICP/AES), gamma energy analysis (GEA), and ion chromatography (IC) analyses, even though only specific analytes are requested. These opportunistic analyses (Kristofzski 1996) should be reported only if no additional preparatory work is required (e.g., running additional standards) and if the error associated with the results is documented. No reruns or additional analyses should be performed to improve recovery for analytes not specifically requested in Tables 1 or 2 . 
No specific requirements for analytical accuracy and precision are presented with respect to the PRSST method. It is understood that the final results of this measurement are strongly subject to interpretation and that accuracy is dependent upon absolute calibration. To that end, accuracy must be maintained through the calibration of heater resistance, time, temperature, pressure, containment volume, and sample weight as described in procedure LT-510-103.

\subsection{ORGANIZATION}

The organization and responsibility of key personnel involved with this tank TX-113 characterization project are listed in Table 4.

Table 4: Tank TX-113 Project Key Personnel List

\begin{tabular}{|l|l|l|}
\hline Responsibility & Organization & lndividual \\
\hline TX-113 Tank Coordinator & $\begin{array}{l}\text { TWRS Process Engineering } \\
\text { (LMHC) }\end{array}$ & D. J. McCain, 373-1023 \\
\hline $\begin{array}{l}\text { 222-S Laboratory Point of } \\
\text { Contact (day shift) }\end{array}$ & Analytical Services (WMH) & D. B. Hardy, 376-4878 \\
\hline $\begin{array}{l}\text { 222-S Laboratory Point of } \\
\text { Contact (off-hours) }\end{array}$ & Analytical Services (WMH) & $\begin{array}{l}\text { 222-S Laboratory Shift } \\
\text { Manager, 373-2435 }\end{array}$ \\
\hline $\begin{array}{l}\text { Single-Shell Tank Farm Point } \\
\text { of Contact }\end{array}$ & Tank Farm Operations & $\begin{array}{l}\text { Single-Shell Tank Farm } \\
\text { Operations Shift Manager, } \\
373-3475\end{array}$ \\
\hline
\end{tabular}

\subsection{DELIVERABLES}

All analyses will be reported as Format I, III, or IV as indicated in Tables 1 and 2. Additional information regarding reporting formats is given in Schreiber (1998).

\subsection{FORMAT I REPORTING}

Tables 1 and 2 contain the notification limits for each analyte. Any results exceeding their notification limits shall be reported via telephone by the 222-S Laboratory Faculty Planning Team to the Single-Shell Tank Farm Operations shift manager as soon as the data are obtained and reviewed by the responsible scientist. This verbal notification must be followed within one hour by electronic notification to the tank farm operations shift manager, the Tank Waste Remediation System (TWRS) Process Engineering Data Assessment and Interpretation manager, and the tank coordinator responsible for the tank. Additional analyses for verification purposes may be contracted between the performing laboratory and the tank coordinator by either a revision to this SAP or by a letter. 


\subsection{FORMAT III REPORTING}

Analyses identified as primary safety screening analyses in Tables 1 and 2 must be completed within 45 calendar days of the receipt of the last sample at the laboratory sample receiving/loading dock. If no safety screening criteria were exceeded, the laboratory shall electronically notify the tank coordinator and shall follow the notification with a letter to the TWRS Process Engineering Data Assessment and Interpretation manager and the tank coordinator confirming work completion. If any analysis results exceeded the safety screening criteria, a letter identifying the results which exceeded the criteria will be issued.

Any secondary safety screening analyses must be completed within 90 calendar days of the receipt of the last sample at the laboratory sample receiving/loading dock. When the secondary analyses are complete, the laboratory shall issue a letter to the TWRS Process Engineering Data Assessment and Interpretation manager and tank coordinator confirming work completion. If any secondary analysis notification limits were exceeded, the results which exceeded the limits shall be identified.

\subsection{FORMAT IV REPORTING}

The format IV report shall be a data package reporting the results of analyses performed and will resemble a regulatory data package without third party validation. The data package should be prepared by tank and include the data for all samples, including (as applicable) composites, segments, subsegments, drainable liquids, and associated blanks taken and analyzed for this sampling event. The recommended reporting format and the raw data that shall be included are given in detail in Section A5.0 of Schreiber (1997b). The data package shall be issued 180 days after the last sample is received at the laboratory. The raw data shall be accessible to the program in accordance with the laboratory's Records Inventory and Disposition Schedule and until the respective waste tank is closed or the waste is treated.

In addition to this data package, an electronic version of the analytical results shall be provided to the Tank Characterization Database representative on the same day that the final data package is issued. The data must be available to the Washington State Department of Ecology within 7 days of release of the data package. The electronic version shall be in the standard electronic format (Bobrowski et al. 1994).

\subsection{CHANGE CONTROL}

Under certain circumstances, it may become necessary for the performing laboratory to make decisions concerning a sample without review of the data by the customer or the Characterization Project. All significant changes (such as DQO additions or analysis of new, additional samples) shall be documented by TWRS Process Engineering via an Engineering Change Notice to this SAP or by a letter. All changes shall also be clearly documented in the final data report. Insignificant changes may be made by the tank or project coordinator by placing a notation in the permanent record (i.e. note change in extrusion log book or memo to file). Significance is determined by the tank coordinator. 


\section{HNF-3039, Rev. 0}

At the request of the Characterization Project, additional analysis of sample material from this characterization project shall be performed following a revision of this SAP or issuance of a letter. 


\subsection{REFERENCES}

Bauer, R. E., 1998, Data Quality Objective to Support Resolution of the Flammable Gas Safety Issue, HNF-SD-WM-DQO-004, Rev. 3-A, Lockheed Martin Hanford Corporation, Richland, Washington.

Board, D. C., 1997, Tank Waste Remediation System, Characterization Project, Quality Policies, HNF-SD-WM-QAPP-025, Rev. 3, Lockheed Martin Hanford Corporation, Richland, Washington.

Bobrowski, S. F., S. J. Harris, and J. D. Schwab, 1994, Standard Electronic Format Specification for Tank Characterization Database Loader, Version 2.0, (memorandum TCD-94-002 to C. S. Haller, dated June 16), Pacific Northwest Laboratory, Richland, Washington.

Brown, T. M., J. W. Hunt, and L. J. Fergestrom, 1998, Tank Characterization Technical Sampling Basis, HNF-SD-WM-TA-164, Rev. 4, Lockheed Martin Hanford Corporation, Richland, Washington.

Cash, R. J., 1997, Cancellation of Total Cyanide Requirements, (letter 2N160-97-012 to J. G. Kristofzski, June 16), DE\&S Hanford, Incorporated, Richland, Washington.

DOE, 1997, Hanford Analytical Services Quality Assurance Requirements Document DOE/RL96-68, Rev. 1, U.S. Department of Energy, Richland Operations Office, Richland, Washington.

Dukelow, G. T., J. W. Hunt, H. Babad, and J. E. Meacham, 1995, Tank Safety Screening Data Quality Objective, WHC-SD-WM-SP-004, Rev. 2, Westinghouse Hanford Company, Richland, Washington.

Hanlon, B. M., 1998, Waste Tank Summary Report for Month Ending April 30; 1998, HNF-EP-0182-121, Lockheed Martin Hanford Corporation, Richland, Washington.

Kristofzski, J. G., 1996, Directions for “Opportunistic Analyses", (memorandum 79400-96-168 to J.H. Baldwin et al., dated September 11), Westinghouse Hanford Company, Richland, Washington.

Lapin, L. L., 1983, Probability and Statistics for Modern Engineering, Brooks/Cole Publishing Company, Monterey, California.

Markel, L. P., 1997, 222-S Laboratory Quality Assurance Plan, HNF-SD-CP-QAPP-016, Rev. 2 , Rust Federal Services of Hanford, Inc., Richland, Washington. 
HNF-3039, Rev. 0

PHMC, 1997, Project Hanford Quality Assurance Program Description, "Nonconformance Control" (Section 3.0, paragraph 3.3), HNF-MP-599, Rev. 0, Fluor Daniel Hanford Company, Richland, Washington.

Place, D. E., Preliminary Tank Characterization Report for Single-Shell Tank 241-TX-113:Best Basis, HNF-SD-WM-ER-716, Rev.0., SGH Eurisys Services Corporation, Richland, Washington.

Schreiber, R. D., 1998, Fiscal Year 1998 Memorandum of Understanding for the TWRS Characterization Project, HNF-2245 Rev. 0, Lockheed Martin Hanford Corporation, Richland, Washington.

Simpson, B. C., and D. J. McCain, 1997, Historical Model Evaluation Data Requirements, HNF-SD-WM-DQO-018, Rev. 2, Lockheed Martin Hanford Corporation, Richland, Washington. 
HNF-3039, Rev. 0

\section{APPENDIX A:}

EXPECTED PHYSICAL PROFILE OF TANK TX-113 CORE SAMPLES 
HNF-3039, Rev. 0

Table A-1: Tank 241-TX-113 Physical Profile Estimate Riser 3

\begin{tabular}{|c|c|c|c|c|}
\hline Segment & Inches & $\begin{array}{l}\text { Elevation Range } \\
\text { (fi MSL) }\end{array}$ & ४Waste Type, & ४ै४ \\
\hline \multirow[b]{2}{*}{1} & 17 & $644.20-645.60$ & & metal tapes under riser \\
\hline & 2 & $644.02-644.20$ & dry, soft saltcake & $\begin{array}{l}\text { limited to good recovery with push } \\
\text { mode, limited recovery with rotary } \\
\text { mode due to N2 purge }\end{array}$ \\
\hline 2 & 19 & $642.44-644.02$ & dry, soft saltcake & $\begin{array}{l}\text { limited to good recovery with push } \\
\text { mode, limited recovery with rotary } \\
\text { mode due to } 2 \text { purge }\end{array}$ \\
\hline 3 & 19 & $640.85-642.44$ & dry, hard saltcake & limited recovery \\
\hline 4 & 19 & $639.27-640.85$ & dry, hard saltcake & limited recovery \\
\hline 5 & 19 & $637.69-639.27$ & dry, hard saltcake & limited recovery \\
\hline 6 & 19 & $636.10-637.69$ & dry, hard saltcake & limited recovery \\
\hline 7 & 19 & $634.52-636.10$ & dry, hard saltcake & limited recovery \\
\hline 8 & 19 & $632.94-634.52$ & dry, hard saltcake & limited recovery \\
\hline 9 & 19 & $631.35-632.94$ & dry, hard saltcake & limited recovery \\
\hline 10 & 19 & $629.77-631.35$ & damp, soft sludge/saltcake mix & $\begin{array}{l}\text { limited to good recovery, saltcake will } \\
\text { look like sludge/saltcake }\end{array}$ \\
\hline \multirow[t]{2}{*}{11} & 0.5 & $629.73-629.77$ & damp, soft sludge/saltcake mix & $\begin{array}{l}\text { limited to good recovery, saltcake will } \\
\text { look like sludge/saltcake }\end{array}$ \\
\hline & 18.5 & $628.19-629.73$ & damp, soft sludge & good recovery expected \\
\hline 12 & 19 & $626.60-628.19$ & damp, soft sludge & good recovery expected \\
\hline 13 & 19 & $625.02-626.60$ & damp, soft sludge & good recovery expected \\
\hline
\end{tabular}

Note: elevations based on inside tank center bottom for Tank $241-\mathrm{TX}-113$ of $624.73 \mathrm{ft}$. MSL

\section{Figure A-1: Tank 241-TX-113 Physical Profile Estimate}

\section{(Riser 3)}

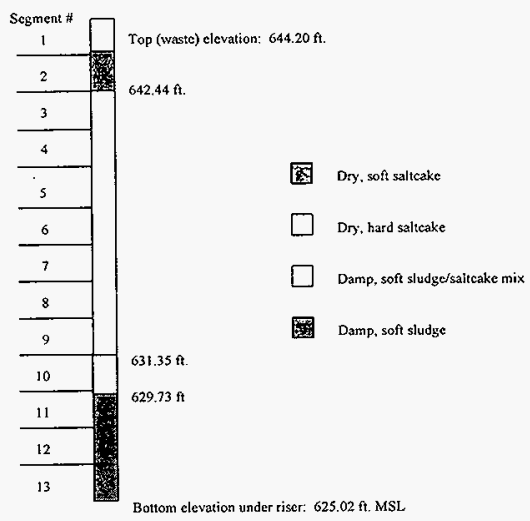


HNF-3039, Rev. 0

Table A-2: Tank 241-TX-113 Physical Profile Estimate

Riser 5

\begin{tabular}{|c|c|c|c|c|}
\hline Segment t & Inches & $\begin{array}{l}\text { Elevation Range } \\
\text { (ft. MSL) }\end{array}$ & Waste Type & (4) Compents \\
\hline \multirow[b]{2}{*}{1} & 16 & $644.20-645.54$ & & metal tapes under riser \\
\hline & 3 & $643.96-644.20$ & dry, soft salttcake & $\begin{array}{l}\text { limited to good recovery with push } \\
\text { mode, limited recovery with rotary } \\
\text { mode due to N2 purge }\end{array}$ \\
\hline 2 & 19 & $642.38-643.96$ & dry, soft saltcake & $\begin{array}{l}\text { limited to good recovery with push } \\
\text { mode, limited recovery with rotary } \\
\text { mode due to N2 purge }\end{array}$ \\
\hline 3 & 19 & $640.79-642.38$ & dry, hard saltcake & limited recovery \\
\hline 4 & 19 & $639.21-640.79$ & dry, hard saltcake & limited recovery \\
\hline 5 & 19 & $637.63-639.21$ & dry, hard saltcake & limited recovery \\
\hline 6 & 19 & $636.04-637.63$ & dry, hard saltcake & limited recovery \\
\hline 7 & 19 & $634.46-636.04$ & dry, hard saltcake & limited recovery \\
\hline 8 & 19 & $632.88-634.46$ & dry, hard saltcake & limited recovery \\
\hline 9 & 19 & $631.29-632.88$ & dry, hard saltcake & limited recovery \\
\hline \multirow{2}{*}{10} & 18 & $629.77-631.29$ & damp, soft sludge/saltcake mix & $\begin{array}{l}\text { limited to good recovery, saltcake will } \\
\text { look like sludge/saltcake }\end{array}$ \\
\hline & 1 & $629.71-629.77$ & damp, soft sludge/saltcake mix & $\begin{array}{l}\text { limited to good recovery, saltcake will } \\
\text { look like sludge/saltcake }\end{array}$ \\
\hline 11 & 19 & $628.13-629.71$ & damp, soft sludge & good recovery expected \\
\hline 12 & 19 & $626.54-628.13$ & damp, soft sludge & good recovery expected \\
\hline 13 & 19 & $624.96-626.54$ & damp, soft sludge & good recovery expected \\
\hline
\end{tabular}

Note: elevations based on inside tank center bottom for Tank $241-T X-113$ of $624.73 \mathrm{ft}$. MSL

Figure A-2: Tank 241-TX-113 Physical Profile Estimate

(Riser 5)

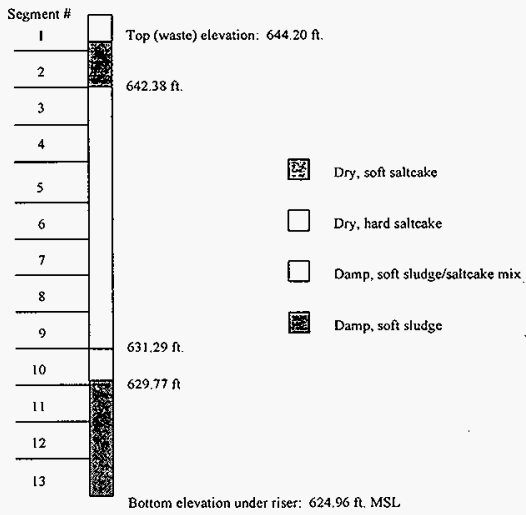




\begin{tabular}{|c|c|c|c|c|c|}
\hline \multirow[b]{2}{*}{ Distribution } & \multirow{2}{*}{\multicolumn{3}{|c|}{$\begin{array}{l}\text { From } \\
\text { Technical Basis and Planning }\end{array}$}} & \multicolumn{2}{|l|}{ Page 1 of 1} \\
\hline & & & & \multicolumn{2}{|c|}{ Date $\quad 07 / 30 / 98$} \\
\hline \multicolumn{4}{|l|}{ Project Title/Work Order } & \multicolumn{2}{|c|}{ EDT No. EDT-622460 } \\
\hline \multicolumn{4}{|c|}{$\begin{array}{l}\text { HNF-3039, Rev, 0, "Tank 241-TX-113 Rotary Mode Core Sampling and } \\
\text { Analysis Plan" }\end{array}$} & \multicolumn{2}{|c|}{ ECN No. N/A } \\
\hline Name & MSIN & $\begin{array}{c}\text { Text } \\
\text { With } \\
\text { All } \\
\text { Attach. }\end{array}$ & Text Only & $\begin{array}{l}\text { Attach./ } \\
\text { Appendix } \\
\text { Only }\end{array}$ & $\begin{array}{l}\text { EDT/ECN } \\
\text { OnTy }\end{array}$ \\
\hline
\end{tabular}

U. S. Department of Energy -

Richland Field Office

C. A. Babel

W. LiOu

J. A. Poppiti

$\begin{array}{ll}\text { S7-54 } & x \\ \text { S7-54 } & x \\ \text { S7-54 } & x\end{array}$

Lockheed Martin Hanford Corp.

D. C. Board

J. G. Burton

R. N. Da7e

K. M. Hal1

W. J. Kennedy

J. S. Lee

D. J. McCain

R. R. Thompson

T.C.S.R.C.

$\begin{array}{ll}\text { S7 }-07 & x \\ \text { S7-21 } & x \\ \text { S7-12 } & x \\ \text { R2-12 } & x \\ \text { S7-03 } & x \\ \text { S7-03 } & x \\ \text { R2-12 } & x \\ \text { R2-12 } & x \\ \text { R1 }-10 & x\end{array}$

Lockheed Martin Services, Inc.

Central Files

B1-07 $\quad X$

Los Alamos Technical Associates

R. W. Lambie

$57-04 \quad x$

Numatec Hanford Corporation

W. I. Winters

T6-50 $\quad X$

Waste Management Federa] Services of Hanford. Inc.

$\begin{array}{lll}\text { R. Akita } & T 6-20 & X \\ \text { R. A. Esch } & T 6-12 & X \\ \text { R. K. Fuller } & T 6-50 & X \\ \text { D. B. Hardy } & T 6-12 & X \\ \text { K. L. Powel1 } & T 6-12 & X \\ \text { F. H. Steen } & T 6-12 & X\end{array}$

